\title{
A Teoria das Representações Sociais nas pesquisas da Enfermagem brasileira
}

\author{
The Social Representations Theory in Brazilian nursing research
}

La Teoría de las Representaciones Sociales en las investigaciones de la Enfermería brasileña

\author{
Sílvio Éder Dias da Silva', Brigido Vizeu Camargo", Maria Itayra Padilha"I \\ ' Universidade Federal do Pará, Faculdade de Enfermagem. Doutorado Interinstitucional \\ Universidade Federal do Parál Universidade Federal de Santa Catarina. Belém-PA, Brasil. \\ " Universidade Federal de Santa Catarina, Departamento de Psicologia, Laboratório de Psicologia Social, \\ da Comunicação e da Cognição. Florianópolis-SC, Brasil. \\ II' Universidade Federal de Santa Catarina, Departamento de Enfermagem, \\ Grupo de Estudos da História do Conhecimento da Enfermagem. Pesquisadora do CNPq. Florianópolis-SC, Brasil.
}

Submissão: 22-10-2010 Aprovação: 13-12-2011

\section{RESUMO}

Esta pesquisa teve como objetivo analisar os aspectos metodológicos nas teses e dissertações que empregaram a teoria das representações sociais TRS na enfermagem brasileira. Foram consideradas 22 teses e 44 dissertações. Empregou-se uma análise de conteúdo de tipo categorial feita com base em cinco aspectos: objetos estudados, lugar da TRS, número de participantes do estudo, técnicas de coleta e de análise dos dados. A predominância de estudos de caso e o uso, sobretudo da entrevista semiestruturada, embora pertinentes para o estudo de RS, não contemplam suficientemente a dimensão do compartilhamento deste tipo de conhecimento na sociedade. Considerar o conhecimento leigo para se compreender os cuidados em relação à saúde contextualiza os estudos sobre a atenção em relação à saúde.

Descritores: Enfermagem; Conhecimento; Psicologia Social.

\section{ABSTRACT}

This study aimed to examine methodological issues in the theses and dissertations that used social representations theory (SRT) in Brazilian nursing. Twenty-two dissertations and 44 theses were considered. Categorical content analysis was applied, based on five aspects: objects studied, place of SRT, the number of study participants, data collection techniques and data analysis. The predominance of case studies and use, especially of semi-structured interviews, although relevant to the study of SR, do not sufficiently consider the extent of suck knowledge sharing in society. One must take into account lay knowledge to understand health care contextualized studies on health care.

Key words: Nursing; Knowledge; Social Psychology.

\section{RESUMEN}

La finalidad de este estudio fue examinar las cuestiones metodológicas en las tesis y disertaciones en que se utilizó la teoría de las representaciones sociales (TRS) en la enfermería brasileña. Fueron consideradas 22 tesis y 44 disertaciones. Fue aplicado el análisis de contenido categórico, basado en cinco aspectos: los objetos estudiados, lugar de TRS, número de participantes, técnicas de recopilación de datos y análisis de datos. El predominio de estudios de caso y el uso, especialmente de la entrevista semi-estructurada, aunque relevantes para el estudio de la RS, no contemplan suficientemente el tamaño del compartir de esos conocimientos en la sociedad. Considerar los conocimientos laicos para entender los cuidados respecto a la salud contextualiza los estudios sobre la atención a la salud.

Palabras clave: Enfermería; Conocimiento; Psicología Social. 


\section{CONSIDERAÇÕES INICIAIS}

A diversidade de enfoques teóricos e metodológicos é uma característica marcante da enfermagem. Dentre estes se destaca a Teoria das Representações Sociais (TRS), que é muito empregada nesta área, devido à possibilidade do pesquisador captar a interpretação dos próprios participantes da realidade que se almeja pesquisar, possibilitando a compreensão das atitudes e comportamentos que um determinado grupo social frente a um objeto psicossocial. Considerando que a representação social favorece conhecer a prática de um determinado grupo, ela permite à enfermagem realizar intervenções que, por respeitarem as características especificas de cada segmento social, serão mais eficientes.

Embora o presente trabalho tenha como foco de análise teses e dissertações que fazem uso da TRS, observa-se também um aumento de publicações deste tipo em revistas científicas. Este fato indica uma decorrência da existência de um número significativo de dissertações e algumas teses defendidas nos anos 2000, que resultaram em artigos, por exigência dos próprios programas de pós-graduação, ampliando assim a visibilidade dos trabalhos que empregam a TRS na enfermagem.

A TRS teve seu início na França, na década de 50, quando o psicólogo social Serge Moscovici buscou entender como a psicanálise, um novo saber especializado, era compreendido pela sociedade francesa naquela época. A partir desse estudo, ele conseguiu compreender como um objeto científico torna-se objeto do senso comum. Este trabalho foi levado publicado em sua obra "A psicanálise, sua imagem e seu público", que lhe concedeu o titulo de "criador da teoria das representações sociais"(1).

As representações sociais visam integrar um fenômeno social não familiar, que, por ser desconhecido, gera medo e ansiedade, ao conhecimento que os indivíduos e grupos dispõem sobre seu cotidiano. Por tal motivo, é necessária a sua assimilação ao referencial conceitual dos indivíduos, para que possa se tornar familiar ${ }^{(2)}$. Isto ocorre também quando se apresenta determinado conhecimento especializado (reificado) a um determinado grupo social, e a partir de sua apresentação ele é reelaborado, tornando-se uma nova forma de conhecimento denominado de consensual, decorrente das conversações e do consenso entre os membros de um determinado grupo social.

As representações sociais são uma forma de conhecimento socialmente organizado e partilhado, que tem objetivo prático e colabora para a constituição de uma realidade comum a um grupo social, podendo ser denominada como saber de senso comum ou ainda saber natural. Esta forma de conhecimento é distinta, entre outras, do conhecimento cientifico. Entretanto, é tida como um objeto de estudo legítimo da própria ciência. Ele apresenta relevância para a vida social já que indica processos cognitivos encarnados nas interações sociais ${ }^{(3)}$.

As representações sociais são reconhecidas como sistemas de interpretação que dirigem nossa relação com o mundo e com os outros. Elas norteiam e estabelecem as condutas e as comunicações sociais. Da mesma forma, elas intervêm em processos variados, tais como difusão e a assimilação dos conhecimentos, o desenvolvimento individual e coletivo, a definição das identidades pessoais e sociais, a expressão de grupos e as transformações sociais ${ }^{(3)}$.

A TRS é abrangente, pois utiliza conceitos sobre atitudes, opiniões, imagens e outros, não os excluindo, mas incorporando-os. A integração desses conceitos favorece compreender a realidade que nos circunda ${ }^{(4)}$. Uma representação social possui duas faces que se estruturam: a figurativa e a significação, pois cada figura está ligada a um sentido e vice-versa, favorecendo a percepção da relação entre o objeto identificado e a sua interpretação ${ }^{(1)}$.

Na esfera social, dentre vários tipos de conhecimento, nos chamam a atenção duas formas: o conhecimento reificado e o consensual. O primeiro se refere ao conhecimento científico e ao especializado e é tido como um saber "autêntico" que não admite contradição. Este tipo de conhecimento é restrito a uma pequena parcela da comunidade - a cientifica ou técnica. Já o segundo tipo diz respeito ao conhecimento não especializado, sendo denominado saber "ingênuo", ou como é conhecido universalmente senso comum, uma forma de saber presente na maioria da população ${ }^{(1)}$. Um aspecto importante desta forma particular de conhecimento é que ela gera atitudes, opiniões e crenças ${ }^{(6)}$.

A TRS reconhece o valor da dimensão subjetiva, o aspecto cognitivo do indivíduo, que segundo esta perspectiva interfere nas práticas sociais, nas atitudes e condutas relativas ao objeto da representação. A pesquisa que usa a teoria das representações sociais como referencial teórico focaliza sua atenção no conhecimento dos participantes da mesma, enquanto conhecimento importante para se compreender o cotidiano dos mesmos. O referencial da TRS permite esclarecer como se dá o processo de assimilação dos fatos que ocorrem no meio, como os mesmos são compreendidos pelos indivíduos e grupos, e como o conhecimento construído sobre estes fatos são expressos por meio de sua comunicação e em seus comportamentos.

\section{OBJETIVO}

O objetivo do presente trabalho é analisar como a TRS vem sendo utilizada na produção do conhecimento da enfermagem brasileira. Para isto pretende-se: identificar as dissertações e teses da enfermagem brasileira que empregaram a TRS; e analisar, através dos resumos, os aspectos metodológicos indicados na execução das mesmas, considerando o período de 1977 a 2007.

\section{METODOLOGIA}

Este estudo consiste numa pesquisa bibliográfica sobre a produção cientifica na enfermagem brasileira que empregou a TRS. A fonte dos documentos para a constituição do corpus de análise foi o Banco de Teses e Dissertações do Centro de Estudos e Pesquisa em Enfermagem (CEPEn) da Associação Brasileira de Enfermagem (ABEn). Primeiramente foi feita uma consulta aos resumos dos trabalhos cujo título indicasse o emprego da TRS. No período considerado, de 1977 a 2007, 
foram produzidas 22 teses e 44 dissertações que constituíram o corpus da análise.

Posteriormente, foi utilizada uma análise de conteúdo categorial dos resumos destes trabalhos, onde as categorias se referiam a aspectos da metodologia empregada nos estudos, a saber: o objeto de estudado, lugar da Teoria das Representações Sociais na pesquisa, número de participantes, técnica de coleta e de análise dos dados ${ }^{(7)}$.

\section{RESULTADOS E DISCUSSÃO}

A área de pesquisa da educação foi pioneira nos estudos com a TRS no Brasil, sendo responsável pela maior produção de trabalhos na Jornada Internacional de Representações Sociais (JIRS) até o ano de 2003. A partir desta época, a área da saúde passou a apresentar o maior número de trabalhos neste tipo de evento científico, embora as duas áreas se equivalham neste aspecto. No entanto quando se trata do foco na TRS, e não no fenômeno, a educação apresenta um número maior de trabalhos que a área da saúde ${ }^{(7)}$. $\mathrm{O}$ aumento do número de trabalhos de enfermagem que utiliza a TRS a partir da JIRS de 2003 foi antecedido pela presença de uma quantidade considerável de trabalhos no sistema de pós-graduação brasileiro no período de 1995 a 2005. Neste período foram defendidas 22 teses e 44 dissertações que empregaram o referencial teórico das representações sociais. Este dado, junto com o dado das JIRS, indica claramente o interesse da enfermagem brasileira pela $\operatorname{TRS}^{(8)}$.

\section{O lugar da TRS nas dissertações e teses de enfermagem}

A análise do conteúdo do resumo destes trabalhos acadêmicos evidenciou os seguintes temas: o cuidado de enfermagem, o processo saúde-doença, assistência de enfermagem, saúde da criança, saúde do adulto e da mulher. São temas de orientação mais pragmática, característica da área da saúde, e particularmente da enfermagem. O interesse das pesquisas é voltado ao conhecimento do senso comum sobre cuidados e assistência ligados atividade da enfermagem.

A TRS tem sido amplamente empregada na área da saúde com a intenção da promoção da qualidade de vida, a utilização de conhecimentos especializados passou a considerar o conhecimento não especializado, o conhecimento leigo de diversos grupos sociais com a intenção de lidar com a complexidade do problema ${ }^{(2)}$.

Nem sempre a pesquisa de enfermagem deixa clara a diferença entre a TRS e o fenômeno das representações sociais. O fenômeno refere-se a teorias do senso comum, que são utilizadas para organizar as práticas sociais presente no cotidiano, além da compreensão que os leigos têm do meio social que estão inseridos. Já a TRS trata de conjecturas sobre este fenômeno, fazendo uso do conhecimento cientifico, isto é, a TRS determina definições e pressuposições para a realização de estudo cientifico das teorias ditas leigas ${ }^{(2)}$.

Um estudo anterior observou-se que a TRS tem sido utilizada como teoria, enquanto método ou ainda como fenômeno a partir da análise dos resumos apresentados nas JIRS ${ }^{(2)}$. Para que uma pesquisa fosse qualificada como pesquisa que efetivamente contemple a TRS, seria necessário satisfazer pelo menos um dos seguintes critérios: menção aos teóricos clássicos que foram responsáveis pela sua gênese e sua difusão respectivamente, Serge Moscovici e Denise Jodelet; ou que apresente o próprio conceito de representação social. Com base neste mesmo critério, no corpus aqui analisado observou-se que apenas 17 das 44 dissertações e 9 das 22 teses empregaram realmente a TRS como marco teórico dos seus estudos.

As razões que levaram os autores de mais de $1 / 3$ das dissertações e quase metade das teses, aqui consideradas, a fazer menção a TRS sem efetivamente esclarecer, nos resumos, o que entendem por esta teoria, na maior parte dos casos deveu-se ao foco no fenômeno das RS. E em uma menor parte dos casos em entender equivocadamente a TRS como uma abordagem metodológica. O foco no fenômeno indica duas possíveis razões para isto: o interesse pragmático em aplicar o conhecimento da pesquisa, e certa deficiência na formação científica dos pesquisadores. Esta última razão, a mais problemática, embora minoritária no material analisado, leva os pesquisadores a considerar a falsa idéia de que a TRS tem seus métodos próprios, e que a partir da teoria e não do objeto da pesquisa que será realizada a seleção dos métodos para o estudo. As representações sociais não se constituem em uma abordagem metodológica; e sim, elas fazem referência a um fenômeno social e a uma teoria cientifica que se propõe explicá-lo ${ }^{(9)}$.

\section{Aspectos propriamente metodológicos}

A caracterização e a estratégia de seleção dos participantes, as técnicas de coleta e as de análise de dados, são três importantes indicadores metodológicos dos trabalhos considerados no corpus de análise desta pesquisa.

Quanto ao número de participantes relatado nos respectivos resumos analisados, verificou-se nas dissertações 16 trabalharam com o número de participantes inferior a 30 indivíduos, 17 com um número superior a 30 e 11 em 11 resumos de dissertações não foi identificado o número de participantes. Quanto às teses: 8 tinham um número de participantes inferior a 30 indivíduos, 6 estudaram mais que 30 indivíduos, enquanto 8 pesquisas não mencionaram o número de indivíduos estudados. Trabalhar com menos de 30 participantes indica que o estudo se aproxima mais do método de estudo de $\operatorname{casos}^{(2)}$. Já com mais de 30 participantes podem se tratar de um levantamento de dados, pois além do número de participantes ser maior não foi mencionada manipulação de variáveis por parte do pesquisador. Estas pesquisas visam caracterizar das representações sociais pelo emprego de questionários e entrevistas ${ }^{(2)}$.

Dentre as técnicas de coleta de dados a que predominou foi a entrevista semiestruturada, seguida pela técnica de livre associação de palavras, grupo focal e a técnica projetiva de desenho livre. Geralmente uma pesquisa utilizava mais de uma técnica de coleta de dados. Estas técnicas objetivavam a triangulação de dados para favorece a captação dos fenômenos das representações sociais(8). A entrevista é uma técnica muito empregada em pesquisas que empregam análises de dados predominantemente qualitativas, essa técnica é muito 
utilizada pelo fato de favorecer a captação de idéias, opiniões e depoimentos que propiciam conhecer o acervo de significados e símbolos que o indivíduo utiliza para se comunicar no seu cotidiano, a sua agenda ${ }^{(10)}$. Entre as diversas modalidades de entrevista nas pesquisas, destacou-se a semiestruturada que consiste aplicação de um roteiro composto de tópicos organizados em uma estrutura inicial que pode ser ampliada com a inserção de novas perguntas. Este tipo de entrevista propicia certo direcionamento para atender aos objetivos da pesquisa $^{(11)}$. Atualmente tem se empregado a denominação semi-dirigida ao invés de semi-estruturada; esta mudança na terminologia se dá pelo fato de que o pesquisador direciona de forma parcial os relatos dos depoentes para os objetivos do estudo, mas com certa flexibilidade que permita ao participante da pesquisa colocar outros aspectos e os articulá-los de forma autônoma ${ }^{(12)}$.

Outra técnica comumente usada nestes trabalhos foi a de livre associação de palavras, que foi aplicada primeiramente por Jung na sua prática clínica com o intuito de realizar um diagnóstico psicológico sobre a estrutura da personalidade de indivíduos ${ }^{(13)}$. Porém, ao contrário de Jung, no campo das representações sociais os pesquisadores visam identificar elementos das representações sociais, através da configuração dos mesmos que constitui a trama ou rede associativa dos conteúdos evocados em relação a cada estímulo indutor ${ }^{(13)}$. A livre associação de palavras por ser uma técnica projetiva os repertórios latentes pertencentes à estrutura psicológica emerge sem passar pelos filtros de censura, um material espontâneo que dificilmente surge durante entrevistas formais. No que se refere à aplicação desta técnica em pesquisas que tratam sobre representações sociais, evidencia-se a importância desta para captação de um conteúdo rico em espontaneidade, livre de contaminação prévia por parte do pesquisador ${ }^{(14)}$. A referida técnica consiste em fornecer palavras-estímulos que estejam de acordo com o objeto de estudo da pesquisa, para que os participantes associem palavras ou expressões que passarem por suas mentes, de uma forma espontânea, a partir da menção do estímulo indutor ${ }^{(14)}$. Este será selecionado de acordo com os critérios de saliência e coerência com os objetivos do estudo em questão.

A técnica dos grupos focais é a terceira mais empregada nas pesquisas que utilizam a teoria das representações sociais. Ela pode ser descrita como uma entrevista fundamentada na interação de um determinado grupo social que está sendo investigado. A interação do grupo favorece a produção de dados e insights que não emergiriam em outra situação(15). Uma grande vantagem desta técnica é que ela propicia uma intensa troca de idéias sobre o objeto estudado, num período de tempo limitado, além de permitir uma discussão dos dados; que contribui para um aprofundamento destes em conjunto ${ }^{(15)}$. Outro ponto interessante para o uso dos grupos focais é que os dados coletados fluem de forma espontânea, livre de contaminações por parte do pesquisador, fato que não ocorre nas entrevistas individuais. Atualmente, no campo das representações sociais, sabe-se que quanto mais espontânea for à informação coletada, mais esta representa o conhecimento consensual do grupo sobre o objeto investigado.
A técnica projetiva de desenho livre consiste em um instrumento de expressão de conteúdos simbólicos de indivíduos inseridos em um determinado grupo social, os desenhos servem como instrumentos de captação da realidade que se planeja investigar, propiciando a realização de inferências a respeito do seu cotidiano ${ }^{(16)}$.

Os instrumentos e os procedimentos específicos que mais têm sido utilizados pela Enfermagem, em suas pesquisas, são as oficinas de criatividade e sensibilidade, a observação, os materiais visuais, as entrevistas, concomitantemente utilizando técnicas de desenhos, modelagens e colagens; tudo isto para captar o processo de construção da face figurativa da RS, que permite ao indivíduo expressar seus pensamentos e seus sentidos, dando-lhe uma concretude, possibilitando a objetificação ${ }^{(8)}$. Já para se captar a identificação da face simbólica da RS, utilizam-se os relatos verbais ou escritos que permite captar as percepções (interpretações) dos sujeitos sobre os conhecimentos a respeito do fenômeno (ancoragem), transformando a imagem em linguagem, dando-lhes uma explicação, um contexto inteligível ao objeto ${ }^{(8)}$.

Quanto às técnicas de análise de dados houve a predominância da técnica de análise de conteúdo, sobretudo a do tipo temática. Esta modalidade de análise trabalha as informações, os temas e a sua significação que se desprende do texto, permitindo sua interpretação sob o enfoque de várias teorias, dentre estas a TRS ${ }^{(17)}$. Esta técnica de análise propicia conhecer uma realidade, por meio das comunicações de indivíduos que tenham vínculos com a mesma. Outro ponto a salientar é que ela é a que mais se ajusta a pesquisa que tem como foco a análise qualitativa dos dados na área da saúde ${ }^{(18)}$. A análise de conteúdo representou na produção de da III Jornada Internacional de Representações Sociais (JIRS) cerca de 30, 4\% do que foi apresentado nos grupos temáticos, o que indica sua prevalência nas pesquisas que fazem uso da $\operatorname{TRS}^{(7)}$.

\section{CONSIDERAÇÕES FINAIS}

Nessa pesquisa constatou-se que a teoria das representações sociais (TRS) tem sido empregada para investigar objetos psicossociais relacionados ao cotidiano do cuidar da enfermagem. E o interesse na saúde focaliza grupos específicos que são priorizados por políticas governamentais de atenção, como, por exemplo, o tema da saúde da mulher. A idéia de considerar os conhecimentos não especializados (as representações sociais) na compreensão do que seja saúde e cuidados com a saúde apresenta um interesse pragmático: fornecer elementos para a implantação ou aprimoramento de serviços de enfermagem.

O corpus analisado, o conjunto de resumos de dissertações e de teses, indica que parte dos estudos, mesmo se autoclassificando como pesquisas de "representações sociais", não consideram propriamente a TRS como marco teórico. Há ainda no campo da saúde, e especificamente na enfermagem, certa confusão, por parte dos pesquisadores do que seja o fenômeno (teorias do senso comum) e a teoria propriamente dita (teorias científicas que tem como objeto de conhecimento as teorias do senso comum). Por outro lado, há a valorização 
em considerar o conhecimento leigo para se compreender os cuidados em relação à saúde, o que amplia e contextualiza os estudos sobre a atenção em relação à saúde.

No que se refere às indicações metodológicas dos resumos, parece haver o predomínio de pesquisas de estudos de casos em detrimento do levantamento de dados mais abrangente e com amostras representativas de subpopulações. O uso da entrevista semiestruturada, seguida pela técnica de livre associação de palavras, o grupo focal e a técnica projetiva de desenho livre; embora pertinentes para a captação das teorias do senso comum sobre objetos importantes para os grupos sociais, carecem da dimensão do compartilhamento deste tipo de conhecimento na sociedade, dimensão só captada por levantamento de nados mais abrangentes. A parte mais vulnerável do método de pesquisa nos trabalhos de enfermagem considerados parece ser o uso predominante de análises de conteúdo temático. Esta técnica, embora útil para o estudo das RS, apresenta problemas em controlar a interferência do pesquisador sobre os participantes.

O número pequeno de estudos considerados, e o foco apenas nos resumos, podem ser compreendidos com vieses deste estudo, porém esta pesquisa exploratória favoreceu uma análise preliminar quanto aos aspectos teóricos e metodológicos utilizados nas pesquisas de enfermagem. Ressalta-se que o conhecimento da enfermagem brasileira vai além de dados publicados nas teses e dissertações. Os dados aqui apresentados indicam a necessidade de se realizar trabalhos mais aprofundados sobre o uso da TRS.

\section{REFERÊNCIAS}

1. Moscovici S. Representação social da psicanálise. Rio de Janeiro: Zahar; 1978.

2. Coelho MS, Silva DMGV, Padilha MIS. Representações sociais do pé diabético para pessoas com diabetes mellitus tipo 2. Rev. Esc. Enferm. USP. [periódico online]2009[citado 22 outubro 2010];43(1):65-71. Disponível em: http://www. scielo.br/pdf/reeusp/v43n1/08.pdf

3. Jodelet $D$, organizador. As representações sociais. Rio de Janeiro: UERJ, 2001.

4. Oliveira F, Werba G. Representações sociais. In: Jacques MGC. Psicologia Social Contemporânea. $4^{\text {a }}$ ed. Petrópolis: Vozes, 2000.

5. Camargo BV, Wachelke JFR, Aguiar A. O desenvolvimento metodológico das pesquisas sobre representações sociais em jornadas internacionais de 1998 a 2005. In. Moreira ASP, Camargo BV. Contribuições para a teoria e o método de estudos das representações sociais. João Pessoa: Editora Universitária da UFPB, 2007. 6. Sá CPA. Construção do objeto de pesquisa em representações sociais. Rio de Janeiro: UERJ, 1998.

6. Arruda A. Pesquisa em representações sociais: a produção em 2003. In. Menin MSDS, Shimizu AM, organizador. Experiências e representação social: questões teóricas e metodológicas. São Paulo: Casa do Psicólogo, 2005.

7. Padilha MICS, Guerreiro DMVS, Coelho MS. Aspectos teórico-metodológicos das representações sociais e seu uso na enfermagem. Online Braz. J. Nurs. [periódico online] 2007[citado 22 outubro 2010]; 6(2). Disponível em: http://www.uff.br/objnursing/index.php/nursing/article/ view/j.1676-4285.2007.601/199

8. Camargo BV. O que o caminho interdisciplinar brasileiro da teoria das representações sociais não favorece? In: Contribuições para a teoria e o método de estudos das representações sociais. João Pessoa: Editora Universitária da UFPB, 2007.

9. Paredes EC. Entrevistas: anotações para pesquisadores iniciantes. In. Menin MSDS, Shimizu AM, organizador. Experiências e representação social: questões teóricas e metodológicas. São Paulo: Casa do Psicólogo, 2005.

10. Triviños ANS. Introdução à pesquisa em ciências sociais: a pesquisa qualitativa em educação. São Paulo: Atlas, 1987.

11. Turato ER. Tratado da metodologia da pesquisa clinico-qualitativa: construção teórica-epistemológica discussão comparada e aplicada nas áreas de saúde e humanas. Petrópolis, Rj: Vozes, 2003.

12. Coutinho MP, Nóbrega SM. O teste de livre associação de palavras. In: Coutinho MP. Representações sociais: abordagem multidisciplinar. João Pessoa: Editora Universitária da UFPB, 2003.

13. Spink MJ, organizador. O conhecimento no cotidiano: as representações sociais na perspectiva da psicologia social. São Paulo: Brasiliense, 1995.

14. Banchs MA. Representaciones sociales en proceso: su análisis a através de grupos focales. In: Moreira ASP, organizador. Perspectivas teórico-metodológicas em representações sociais. João Pessoa: UFPB/ Editora Universitária, 2005.

15. Filho EAS. Representações sociais da sala de aula através de desenhos de estudantes do ensino fundamental, público e privado no Rio de Janeiro. In. Contribuições para a teoria e o método de estudos das representações sociais. João Pessoa: Editora Universitária da UFPB, 2007.

16. Bardin L. Análise de conteúdo. Lisboa: Edições 70, 1977.

17. Minayo MCS. O desafio do conhecimento: pesquisa qualitativa em saúde. $10^{a}$ ed. São Paulo: HUCITEC-ABRASCO, 2007. 